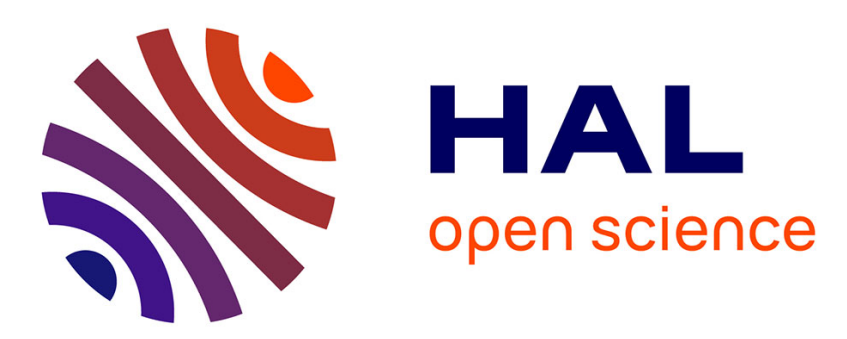

\title{
Critical evaluation of three hemodynamic models for the numerical simulation of intra-stent flows
}

Fatiha Chabi, Stéphane Champmartin, Christophe Sarraf, Ricardo Noguera

\section{To cite this version:}

Fatiha Chabi, Stéphane Champmartin, Christophe Sarraf, Ricardo Noguera. Critical evaluation of three hemodynamic models for the numerical simulation of intra-stent flows. Journal of Biomechanics, 2015, 48 (10), pp.1769-1776. 10.1016/j.jbiomech.2015.05.011 . hal-02486064

\section{HAL Id: hal-02486064 \\ https://hal.science/hal-02486064}

Submitted on 20 Feb 2020

HAL is a multi-disciplinary open access archive for the deposit and dissemination of scientific research documents, whether they are published or not. The documents may come from teaching and research institutions in France or abroad, or from public or private research centers.
L'archive ouverte pluridisciplinaire HAL, est destinée au dépôt et à la diffusion de documents scientifiques de niveau recherche, publiés ou non, émanant des établissements d'enseignement et de recherche français ou étrangers, des laboratoires publics ou privés. 


\title{
Critical evaluation of three hemodynamic models for the numerical simulation of intra-stent flows
}

\author{
Fatiha Chabi ${ }^{a}$, Stéphane Champmartin ${ }^{\text {b,* }}$, Christophe Sarraf ${ }^{a}$, Ricardo Noguera ${ }^{a}$ \\ a ENSAM PARIS, 151 bd de l'Hôpital, 75013 Paris, France \\ ${ }^{\mathrm{b}}$ ENSAM ANGERS, 2 bd du Ronceray, 49035 Angers, France
}

Keywords:

Intra-stent flow

Restenosis

Hemodynamic models

Left coronary artery

Computational fluid mechanics

\section{A B S T R A C T}

We evaluate here three hemodynamic models used for the numerical simulation of bare and stented artery flows. We focus on two flow features responsible for intra-stent restenosis: the wall shear stress and the re-circulation lengths around a stent. The studied models are the Poiseuille profile, the simplified pulsatile profile and the complete pulsatile profile based on the analysis of Womersley. The flow rate of blood in a human left coronary artery is considered to compute the velocity profiles. "Ansys Fluent 14.5" is used to solve the Navier-Stokes and continuity equations. As expected our results show that the Poiseuille profile is questionable to simulate the complex flow dynamics involved in intra-stent restenosis. Both pulsatile models give similar results close to the strut but diverge far from it. However, the computational time for the complete pulsatile model is five times that of the simplified pulsatile model. Considering the additional "cost" for the complete model, we recommend using the simplified pulsatile model for future intra-stent flow simulations.

\section{Introduction}

According to the World Health Organization, 29\% of the 56 million deaths worldwide in 2001 could be attributed to cardiovascular diseases (Murray et al., 2002). They are often due to a decrease in the artery diameter called stenosis related to the growth of atherosclerosis plaque. One of the therapeutic means of treatment is the stent implementation. However intra-stent restenosis often occurs in bare stents (Williams et al., 2000; Mehran et al. 1999a,b). To solve this problem, drug-eluting stents have been designed since the 2000s with encouraging results but with new risks such as late thrombosis (Kastrati et al., 2007). Intra-stent restenosis is a multifaceted phenomenon: besides human factors, it depends on how the surgeon implants the stent, on its shape, on the drug used for an eluting stent, etc. It is also strongly coupled with the blood flow dynamics near the stent struts. LaDisa et al. (2005) or Wentzel et al. (2001) show that low wall shear stresses promote cell proliferation and neo-intimal hyperplasia. This occurs in re-circulation zones induced by the flow separation around the stent strut. Therefore it is essential to characterize properly the dynamics of in-stent blood flows. In most approaches found in the literature, the blood flow is modeled either as a Poiseuille profile (Bozsak et al., 2014; Chen et al., 2014; Weiler et al., 2012) although

\footnotetext{
* Corresponding author. Tel.: +33 241207 341; fax: + 33241207362 .

E-mail address: stephane.champmartin@ensam.eu (S. Champmartin).
}

it seems that this model is not adequate to accurately calculate the characteristics of the arterial flows or as a parabolic profile with an unsteady maximum velocity (Seo et al., 2005; Barakat and Cheng, 2000; Kim et al., 2004). In this work, we propose a more physically acceptable model for the blood flow and we compare it with the simplified models for the flow in the human left coronary artery. In the following section, the simplified hemodynamic models and the alternative model based on the work of Womersley (1955) are exposed.

\section{Hemodynamic models}

Modeling blood flows is very challenging: blood is a non-Newtonian fluid, the vessels are elastic, the flows are pulsatile and possibly turbulent. It is almost impossible to consider all these characteristics at once and simplifications are required. The nonNewtonian characteristics of blood generally occur in small vessels for low values of the shear rate $\dot{\gamma}<100 \mathrm{~s}^{-1}$ (Merrill, 1969) and of the Womersley number $\alpha<1$ (McDonald, 1955). In this work the Womersley number is $\alpha=2.7$ and the shear rate can be estimated as $\dot{\gamma}=114 \mathrm{~s}^{-1}$ in the most critical case. Therefore blood can be considered as a Newtonian fluid. The elasticity of the vascular walls affects the pressure and the velocity field but when the subject becomes older and ill, the arterial rigidity increases. Ozolanta et al. (1998) report that the elastic modulus of the coronary arteries increases from 1.2 MPa (0-1 year) to $4.1 \mathrm{MPa}$ (60-80 years). 
Likewise, patients with hypercholesterolemia, hypertension and atherosclerosis present stiffer vessels. Grosse et al. (2008) and recently Pielhop et al. (2015) compare the flow fields for rigid and elastic walls to test the Womersley model. They show that the rigid and elastic models converge when the Womersley and Reynolds numbers are low enough. Knowing that these parameters are still smaller in our study than in theirs, we consider that the effects of elasticity are small and that the vessel has a constant circular crosssection. Finally the Reynolds number is low and the flow is laminar (McDonald, 1955). Some of these assumptions are debatable but the main objective here is more the qualitative evaluation of some commonly used hemodynamic models in CFD rather than the reproduction of the complex details of real arterial flows. We now present some of the models found in the literature, which are based on the Poiseuille profile, and develop another approach to model the blood flow.

\subsection{Existing simplified models}

The models found in the literature for intra-stent flows can be divided into steady and unsteady models. The simplest way to model the stationary laminar flow of a Newtonian fluid of dynamic viscosity $\mu$ and density $\rho$ in a tube of radius $R$ subject to a pressure gradient $\Delta P / L$, is the Poiseuille parabolic profile:

$u(r)=\frac{\Delta P R^{2}}{4 \mu L}\left(1-\frac{r^{2}}{R^{2}}\right)=2 V\left(1-\frac{r^{2}}{R^{2}}\right)$

with $V$ being the average velocity. We refer to this model in the following lines as the PM (Poiseuille Model). It is accepted that it remains stable for Reynolds numbers $R e=\rho V 2 R / \mu \leq 2200$. The flow rate is

$Q=\pi R^{2} V=\int_{0}^{R} u(r) \times 2 \pi r d r=\frac{\pi R^{4} \Delta P}{8 \mu L}$

The wall shear stress is

$\tau_{w}=\mu \frac{d u}{d r} r_{r=R}=-\frac{\Delta P R}{2 L}=-\frac{4 \mu V}{R}$

The drawback of this model is that it cannot account for the unsteady character of real physiological flows. In recent studies (Coppola and Caro, 2009; O'Brien et al., 2013; Kolachalama et al., 2009) a simplified pulsatile model is used

$u(r, t)=2 V(t)\left(1-\frac{r^{2}}{R^{2}}\right)$

with $V(t)$ being the instantaneous average velocity. The expressions of $Q(t)$ and of $\tau_{w}(t)$ are identical to Eqs. (2.2) and (2.3) with $V$ $(t)$ instead of $V$. Eq. ((2.4) is not a solution of the Navier-Stokes equations (except at very low frequencies) and it misrepresents the flow when pulsatile effects are important because the profiles are no longer parabolic and present a phase shift between $Q$ and $\Delta P / L$. In the next parts, this model is referred to as SPM (Simplified Pulsatile Model).

\subsection{A more physically acceptable model}

Womersley (1955) solved the unsteady Stokes equation for the flow in a rigid circular vessel: $u(r, t)=\Re\left\{\frac{\tilde{a}}{i \rho \omega}\left(1-\frac{J_{0}\left(i^{3 / 2} \alpha r / R\right)}{J_{0}\left(i^{3 / 2} \alpha\right)}\right) e^{i \omega t}\right\}$

subject to the pressure gradient:

$-\frac{\partial P}{\partial z}=\mathfrak{R}\left\{\tilde{a} e^{i \omega t}\right\}$

In these expressions, $\mathfrak{R}$ is the real part of a complex expression and $J_{0}$ is the Bessel function of the first kind and of zero order. The Womersley number $\alpha=R \sqrt{\rho \omega / \mu}$ accounts for the pulsatility of the flow. The unsteady flow rate is

$Q(t)=\Re\left\{\frac{\pi R^{2} \tilde{a}}{i \rho \omega}\left(1-\frac{2 J_{1}\left(i^{3 / 2} \alpha\right)}{\alpha i^{3 / 2} J_{0}\left(i^{3 / 2} \alpha\right)}\right) e^{i \omega t}\right\}$

with $J_{1}$ being the Bessel function of the first kind and order 1 . The wall shear stress is

$\tau_{w}=\Re\left\{\frac{\tilde{a} R i^{1 / 2}}{\alpha} \frac{J_{1}\left(i^{3 / 2} \alpha\right)}{J_{0}\left(i^{3 / 2} \alpha\right)} e^{i \omega t}\right\}$

The pressure gradient for real blood flows being a periodic waveform, it can be written as the sum of terms of the form of Eq. (2.6):

$-\frac{\partial P}{\partial z}=\Re\left\{\sum_{n=0}^{\infty} \tilde{a}_{n} e^{i n \omega t}\right\}=a_{0}+\Re\left\{\sum_{n=1}^{\infty} \tilde{a}_{n} e^{i n \omega t}\right\}$

with $\tilde{a}_{n}=A_{n}-i B_{n}$ being the complex amplitude of the $n$th harmonics of the pressure gradient $\left(A_{n}\right.$ and $B_{n}$ are real with $\tilde{a}_{0}=A_{0}$ and $B_{0}=0$ ). For a pressure gradient like Eq. (2.9), the velocity profile is the superposition of solutions such as Eq. (2.5) because of linearity of the Stokes equation:

$$
\begin{aligned}
u(r, t)= & \frac{A_{0} R^{2}}{4 \mu}\left(1-\frac{r^{2}}{R^{2}}\right) \\
& +\Re\left\{\sum_{n=1}^{\infty} \frac{\tilde{a}_{n}}{i \rho n \omega}\left(1-\frac{J_{0}\left(i^{3 / 2} \alpha_{n} r / R\right)}{J_{0}\left(i^{3 / 2} \alpha_{n}\right)}\right) e^{i n \omega t}\right\}
\end{aligned}
$$

with $\alpha_{n}=R \sqrt{n \rho \omega / \mu}$ being the generalized Womersley number. The flow rate is

$Q(t)=\frac{A_{0} \pi R^{4}}{8 \mu}+\Re\left\{\sum_{n=1}^{\infty} \frac{\pi R^{2} \tilde{a}_{n}}{i \rho n \omega}\left(1-\frac{2 J_{1}\left(i^{3 / 2} \alpha_{n}\right)}{i^{3 / 2} \alpha_{n} J_{0}\left(i^{3 / 2} \alpha_{n}\right)}\right) e^{i n \omega t}\right\}$

and the wall shear stress:

$\tau_{W}(t)=\frac{A_{0} R}{2}+\Re\left\{\sum_{i=1}^{\infty} \frac{\tilde{a}_{n} R i^{1 / 2}}{\alpha_{n}} \frac{J_{1}\left(i^{3 / 2} \alpha_{n}\right)}{J_{0}\left(i^{3 / 2} \alpha_{n}\right)} e^{i n \omega t}\right\}$

The difficulty in this model is to obtain the real and imaginary parts of $J_{0}$ and $J_{1}$. This separation involves the Kelvin functions ber and $b e i_{n}$ :

$J_{n}\left(i^{3 / 2} x\right)=\operatorname{ber}_{n}(x)+i b e i_{n}(x)$

Moreover the application of this model to actual blood flows requires the measurement of the pressure gradient which cannot be obtained easily. It is much simpler to measure the flow rate, for 
instance by a Doppler ultrasound flowmeter. We have therefore adapted Eq. (2.10) in the case where $Q(t)$ or $V(t)=Q(t) / \pi R^{2}$ is known. Since the signal is periodic with period $T=2 \pi / \omega$, it can be decomposed into Fourier series:

$V(t)=V_{0}+\sum_{n=1}^{\infty} V_{n} \cos (n \omega t)+W_{n} \sin (n \omega t)$

The coefficients $V_{n}$ and $W_{n}$ are such that

$V_{0}=\frac{1}{T} \int_{0}^{T} V(t) d t$ and $W_{0}=0$ for $n=0$

$V_{n}=\frac{2}{T} \int_{0}^{T} V(t) \cos (n \omega t) d t$ and $W_{n}$

$=\frac{2}{T} \int_{0}^{T} V(t) \sin (n \omega t) d t$ for $n$

$\geq 1$

The knowledge of $V_{n}$ and $W_{n}$ enables us to calculate $A_{n}$ and $B_{n}$ :

$A_{0}=\frac{8 \mu V_{0}}{R^{2}}$ and $B_{0}=0$ for $n=0$

$A_{n}=\frac{R_{n} V_{n}+P_{n} W_{n}}{R_{n}^{2}+P_{n}^{2}} \quad$ and $\quad B_{n}=\frac{R_{n} W_{n}-P_{n} V_{n}}{R_{n}^{2}+P_{n}^{2}} \quad$ for $n \geq 1$

with

$$
\begin{aligned}
R_{n}= & \frac{2}{\rho n \omega} \\
& \left\{\frac{\operatorname{ber}\left(\alpha_{n}\right)\left[\operatorname{ber}\left(\alpha_{n}\right)+\operatorname{bei}_{1}\left(\alpha_{n}\right)\right]-\operatorname{bei}_{0}\left(\alpha_{n}\right)\left[\operatorname{ber}_{1}\left(\alpha_{n}\right)-\operatorname{bei}_{1}\left(\alpha_{n}\right)\right]}{\left.\alpha_{n} \sqrt{2}\left[\operatorname{ber}_{0}^{2}\left(\alpha_{n}\right)\right]+\operatorname{bei}_{0}^{2}\left(\alpha_{n}\right)\right]}\right\}
\end{aligned}
$$

and

$$
\begin{aligned}
P_{n}= & \frac{1}{\rho n \omega} \\
& \left\{1+2 \frac{\operatorname{ber}_{0}\left(\alpha_{n}\right)\left[\operatorname{ber}_{1}\left(\alpha_{n}\right)-\operatorname{bei}\left(\alpha_{n}\right)\right]+\operatorname{bei}_{0}\left(\alpha_{n}\right)\left[\operatorname{ber}_{1}\left(\alpha_{n}\right)+\operatorname{bei}\left(\alpha_{n}\right)\right]}{\left.\alpha_{n} \sqrt{2}\left[\operatorname{ber} \gamma^{2}\left(\alpha_{n}\right)\right]+\operatorname{bei}^{2}\left(\alpha_{n}\right)\right]}\right\}
\end{aligned}
$$

Finally the velocity profile can be written as

$$
\begin{aligned}
u(r, t)= & \frac{A_{0} R^{2}}{4 \mu}\left(1-\frac{r^{2}}{R^{2}}\right) \\
& +\sum_{n=1}^{\infty}\left[A_{n} \sin (n \omega t)-B_{n} \cos (n \omega t)\right] \\
& {\left[1-\frac{\operatorname{ber}_{0}\left(\alpha_{n}\right) b e r_{0}\left(\alpha_{n} r / R\right)+b e i_{0}\left(\alpha_{n}\right) b e i_{0}\left(\alpha_{n} r / R\right)}{\operatorname{ber}_{0}^{2}\left(\alpha_{n}\right)+b e i_{0}^{2}\left(\alpha_{n}\right)}\right] } \\
& +\left[A_{n} \cos (n \omega t)+B_{n} \sin (n \omega t)\right] \\
& {\left[\frac{b e r_{0}\left(\alpha_{n}\right) b e i_{0}\left(\alpha_{n} r / R\right)-b e i_{0}\left(\alpha_{n}\right) \operatorname{ber}_{0}\left(\alpha_{n} r / R\right)}{\operatorname{ber}_{0}^{2}\left(\alpha_{n}\right)+b e i_{0}^{2}\left(\alpha_{n}\right)}\right] }
\end{aligned}
$$

We can calculate the Kelvin functions from their integral formulation (Watson, 1995):

$$
\begin{aligned}
& \operatorname{ber}_{0}\left(\alpha_{n} \frac{r}{R}\right)=\frac{1}{\pi} \int_{0}^{\pi} \cos \left(\frac{\alpha_{n} \sqrt{2}}{2} \frac{r}{R} \sin x\right) \cosh \left(\frac{\alpha_{n} \sqrt{2}}{2} \frac{r}{R} \sin x\right) d x \\
& \text { bei }_{0}\left(\alpha_{n} \frac{r}{R}\right)=\frac{1}{\pi} \int_{0}^{\pi} \sin \left(\frac{\alpha_{n} \sqrt{2}}{2} \frac{r}{R} \sin x\right) \sinh \left(\frac{\alpha_{n} \sqrt{2}}{2} \frac{r}{R} \sin x\right) d x \\
& \operatorname{ber}_{1}\left(\alpha_{n}\right)=\frac{1}{\pi} \int_{0}^{\pi} \cos \left(x+\frac{\alpha_{n} \sqrt{2}}{2} \sin x\right) \cosh \left(\frac{\alpha_{n} \sqrt{2}}{2} \sin x\right) d x
\end{aligned}
$$

$\operatorname{bei}_{1}\left(\alpha_{n}\right)=\frac{1}{\pi} \int_{0}^{\pi} \sin \left(x+\frac{\alpha_{n} \sqrt{2}}{2} \sin x\right) \sinh \left(\frac{\alpha_{n} \sqrt{2}}{2} \sin x\right) d x$

We refer to this model as the CPM (Complete Pulsatile Model) and in the following section, we apply and compare the three previous models.

\section{Application: flow in the human left coronary artery}

\subsection{Restitution of the flow}

We consider here the flow in a human left coronary artery. This vessel is taken as a circular pipe of constant radius $R=1.75 \mathrm{~mm}$. The dynamic viscosity of the blood is equal to $\mu=3.46 \mathrm{mPa}$ and its density is $\rho=1050 \mathrm{~kg} / \mathrm{m}^{3}$. The instantaneous average velocity $V$ $(t)$ extracted from Bénard (2005) is presented in Fig. 1 (this curve is based on in vivo Doppler measurements of the blood flow in a human left coronary artery).

The period of this signal $T=0.8 \mathrm{~s}$ corresponds to the heartbeat of a person at rest. The Reynolds number ranges between $106<\operatorname{Re}<266$ (laminar regime). This signal is divided into $N=100$ samples $V_{k},(0 \leq k \leq N-1)$ at constant time intervals $\Delta t=T / N$. A discrete Fourier transform is applied to the sampled signal $V_{k}$ to extract the values of $V_{n}$ and $W_{n}$ :

$V_{n}=\frac{2}{N} \sum_{k=0}^{N-1} V_{k} \cos (n \omega k \Delta t)$

$W_{n}=\frac{2}{N} \sum_{k=0}^{N-1} V_{k} \sin (n \omega k \Delta t)$

To reconstruct the initial signal with a sufficient accuracy, 11 terms in Eq. (2.14) are required. Once $V_{n}$ and $W_{n}$ are known, we calculate the velocity profile Eq. (2.21). In the following section, a bare and a stented artery are considered. In the first case, the equations for the velocity profiles, the flow rates and the wall shear stresses are known theoretically but in the second case a numerical procedure is required. In order to validate these models (in all the domain for the bare artery and at the inlet/outlet for the stented artery), we performed a successful comparison of the analytical solutions calculated by the Wolfram Mathematica package with the numerical solutions computed by “ANSYS Fluent 14.5”.

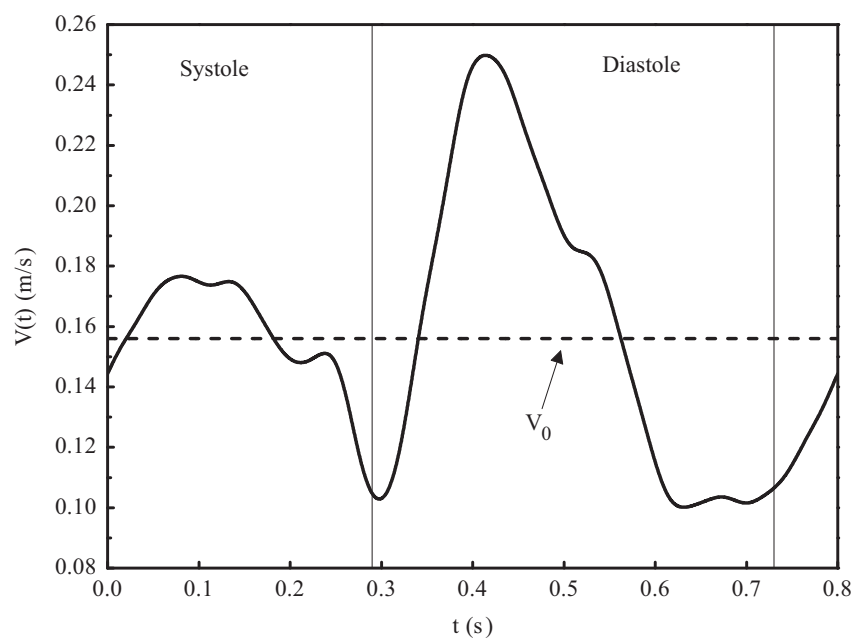

Fig. 1. Mean velocity in the left coronary artery (Bénard, 2005). 


\subsection{Numerical approach}

We restrict our attention to the flow around a single 2D strut of squared cross-section with side $a=0.1 \mathrm{~mm}$. The Navier-Stokes and continuity equations are solved with "ANSYS Fluent 14.5". The flow is axisymmetric around the $z$-axis and in the $(r, z)$ plane, the domain consists of a rectangular surface of radial extension $R=1.75 \mathrm{~mm}$ deprived of the stent cross-section (Fig. 2). We achieved a parametric study of the sensitivity to the mesh, to the size of the domain and to the time step. The solution is found to be independent of the domain size when its extensions upstream and downstream of the strut are respectively $40 a$ and $60 a$. A structured mesh with variable grid size allows a local refinement near the stent while maintaining a reasonable number of cells (a range of 35,000 was used). Finally, for the time step $\Delta t=10^{-3} \mathrm{~s}$, the solution is found to be accurately discretized in time. The convergence criteria are chosen as $10^{-10}$ for each equation and the solutions are considered after the fourth period to ensure the accuracy of the numerical results. The boundary conditions are given in Fig. 2. The velocity profiles for the inlet and outlet are programmed with a UDF (User Defined Function) in C language. In the case of the CPM, the integrals defining $((2.22)-(2.25))$ are calculated with the trapezoid rule (Press et al., 2007). In the following section, the three models are analyzed and compared for a bare artery and a stented artery.

\section{Results and discussion}

\subsection{Bare artery}

For the present flow, the Womersley number $\alpha=R \sqrt{\omega \rho / \mu}=2.7$ is in the intermediate range. It is expected that the pulsatility is significant and that the PM is unsuitable to model the flow. Fig. 3 shows the velocity profiles given by the three hemodynamic models at $t=0, T / 4, T / 2$ and $3 T / 4$ for the SPM and CPM. We note that the pulsatility of the flow is indeed not negligible and that at the intermediate value $\alpha=2.7$, the PM represents a very rough approximation of the flow, especially during the diastole. The wall shear stress is thus undoubtedly underestimated or overestimated during the pulsation. The systole takes place when $0 \mathrm{~s}<t<0.29 \mathrm{~s}$ and $0.73 \mathrm{~s}<t<0.8 \mathrm{~s}$ and the PM is less defective in this phase. Consequently the PM is only an acceptable model during less than one half of the pulsation, mainly during the systole, but fails when

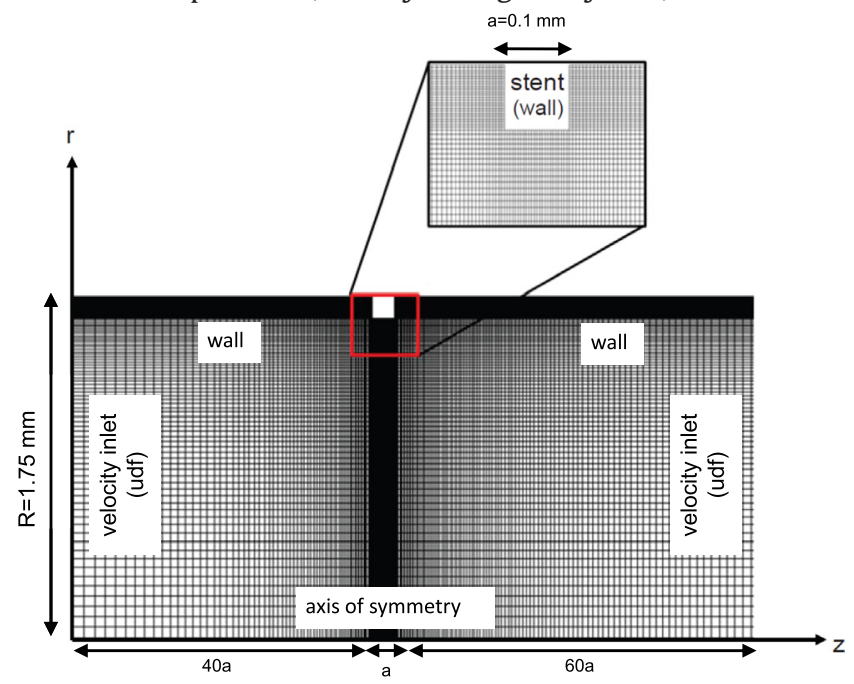

Fig. 2. Schematic of the computational domain of a stented artery and boundary conditions. the blood acceleration and deceleration become large during the diastole. Now comparing both the SPM and the CPM, the difference is lesser but still not negligible. If the CPM gives profiles with a maximum velocity in the axis, these flows are no longer parabolic contrary to the SPM given by Eq. (2.4). We note for example at $t=T / 2$ that the SPM overestimates the velocity in the axis and underestimates it near the wall. The reverse phenomenon occurs at $t=3 T / 4$ with a direct impact on the wall shear stress. Like the PM, the SPM is less defective during the systole and worse during the diastole where large changes in the velocity occur. The wall shear stress is plotted in Fig. 4. As expected from the velocity profiles, the PM gives a poor estimate of the wall shear stress during most of the pulsation. The relative difference between this model and the CPM exceeds $100 \%$ at $t=0.3 \mathrm{~s}$ and $t=0.6 \mathrm{~s}$ (beginning and the end of the diastole). Compared to the PM, the SPM improves the value of the wall shear stress (the time shift between the CPM and the SPM occurs because the pressure gradient and the flow-rate are in phase in the SPM but not in the CPM). There are also significant differences in the amplitude of the wall shear stress: for the SPM, it varies in the range of $0.79 \mathrm{~Pa} \leq \tau_{w} \leq 1.98 \mathrm{~Pa}$, whereas for CPM it ranges at an interval of $0.57 \mathrm{~Pa} \leq \tau_{w} \leq 2.28 \mathrm{~Pa}$. The relative difference between the SPM and the CPM is at most of about $40 \%$, at times near $t=0.3 \mathrm{~s}$ and $t=0.6 \mathrm{~s}$ corresponding again to the beginning and the end of the diastole. During one third of the period, the relative difference between the wall shear stress

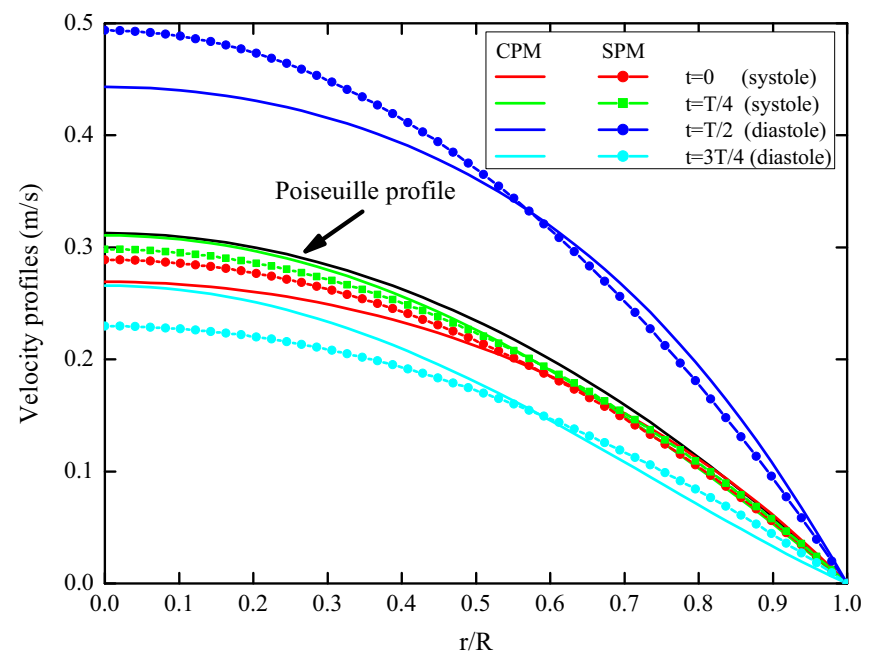

Fig. 3. Comparison of the velocity profiles for three models at different times.

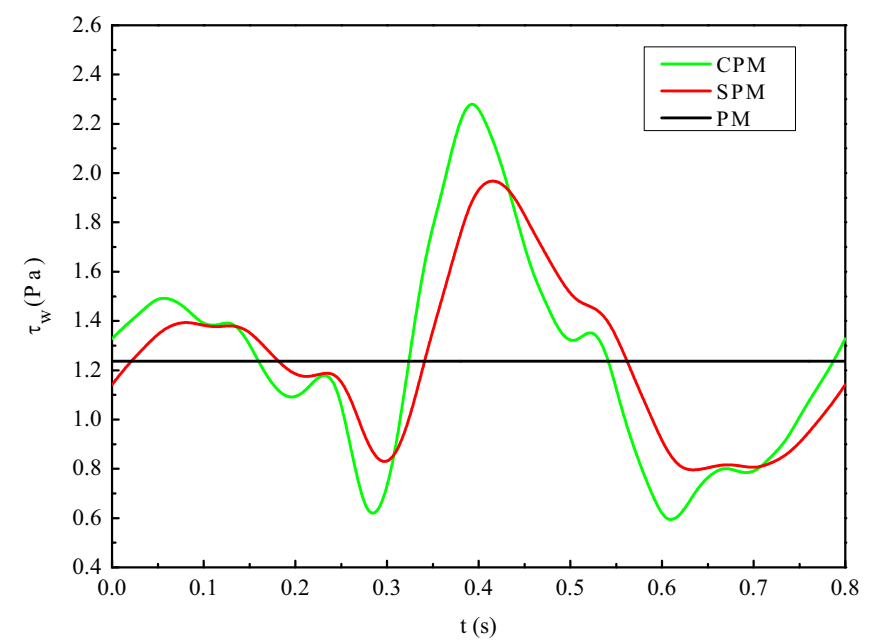

Fig. 4. Wall shear stress for the three models. 
given by the SPM and by the CPM exceeds $10 \%$. It is therefore clear that the choice of the hemodynamic model is very important if we want to estimate at best the shear stress at the cardiovascular wall. We recall here that there is a strong correlation between low values of the shear stress and the development of the atherosclerosis plaque (Bénard, 2005).

\subsection{Stented artery}

Intra-stent flows are characterized by re-circulation zones located upstream and downstream of the strut. These cells are due to the separation of the main flow in the right-angled corners formed by the strut and the artery wall. Inside these re-circulations, low velocities are observed which induce very low values of the wall shear stress. It is also established that the re-circulation cells affect the mass transport in the case of drug-eluting stents (Kolachalama et al., 2009). The size and intensity of these patterns influence the drug delivery in a complex manner by disturbing the mass boundary layer in the vicinity of the stent. It is therefore important to characterize correctly the intra-stent flows and particularly these re-circulation zones. Using dimensional analysis, it is easy to show that the re-circulation length $L$ is a function of the Reynolds number $R e=\rho V 2 R / \mu$ and of some geometric ratio $\Lambda=R / a$. There are many studies related to the relationship between $L$ and $\Lambda$ but most are devoted to low values of $\Lambda$ and to the distal re-circulation cell. In our case, $\Lambda=17.5$ and the comparison with the existing studies is difficult. Let $L_{p}$ and $L_{d}$ be the recirculation lengths in the proximal and distal positions respectively (Fig. 5). In our calculations, $L_{p}$ and $L_{d}$ are determined by searching for the position where the wall shear stress is zero. First we characterize the re-circulation cells in the steady case for comparisons with data available in the literature. Then we complete the study with the time-dependent flows.

\section{Steady flow}

Using the PM, we studied $L_{p}$ and $L_{d}$ in the range $100<R e<270$ (these limits correspond to the minimum and maximum average velocities of the signal in Fig. 1). Fig. 6 shows $L_{p} / a$ and $L_{d} / a$ as a function of Re. We see that these lengths are of the order of the stent size $L_{p, d} \approx a$. Moreover, $L_{d}>L_{p}$ and the distal re-circulation length is about twice the proximal one $\left(1.5<L_{d} / L_{p}<2.3\right)$, indicating that the hydrodynamical regime is slightly inertial. $L_{d}$ increases linearly with $R e$ in accordance with the works dealing with re-circulations behind steps in laminar flow (Armaly et al., 1983). Concerning $L_{p}$, its value decreases as Re increases. We note that $L_{d}$ varies more rapidly with $R e$ than $L_{p}$ : between $R e=100$ and $R e=270$, the relative increase in $L_{d}$ is about $30 \%$ whereas the relative decrease in $L_{p}$ is only about $14 \%$. This can be also seen by calculating:

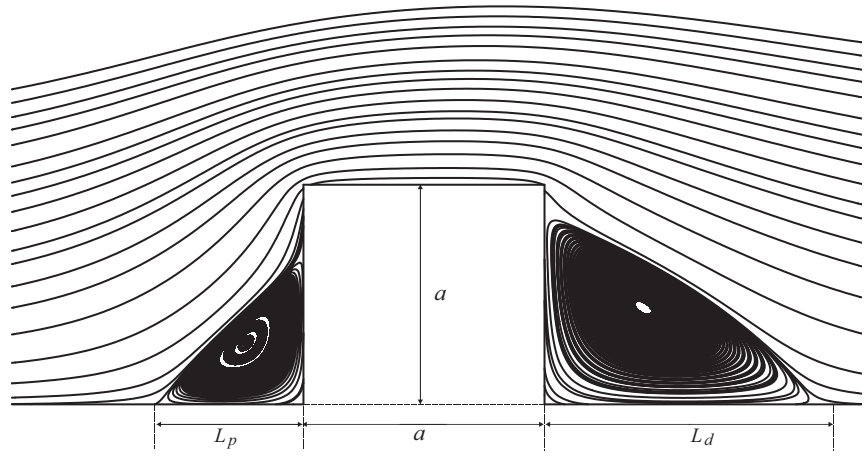

Fig. 5. Streamlines around the stent and definition of $L_{p}$ and $L_{d}$.
$\frac{L_{d, \max }-L_{d, \min }}{L_{p, \max }-L_{p, \min }} \approx 2,8$

The variation of $L_{d}$ is almost thrice the variation of $L_{p}$. This is due to a blockage effect caused by the strut wall in contact with the proximal re-circulation which prevents this cell from contracting and stretching easily. The most similar study can be found in the work of Bénard (2005) who calculated $L_{p}$ and $L_{d}$ for the same ratio $\Lambda=17.5$ and for $R e=106$ and $R e=265$. Kolachalama et al. (2009) also studied the flow around a strut with a square cross-section, an aspect ratio $\Lambda=15$ slightly lower than ours, and for $R e=141$ and $R e=282$. Table 1 compares our results with these studies. A good agreement is found between our values of $L_{p}$ and $L_{d}$ and those of Bénard (2005) with relative differences of less than 6\%. The comparison with Kolachalama et al. (2009) is less satisfactory: his values of the re-circulation lengths are substantially smaller than ours on both sides of the stent. This may be due to the difference in $\Lambda$ but it is unlikely that such a low change in this parameter could produce such large effects. In a more qualitative way, Seo et al. (2005) studied $L_{d}$ for a semi-circular stent. For $R e=200$ and for $\Lambda=20$, they obtained $L_{d} \sim a$ despite the difference in the shape of the stent. Oliveira et al. (2007) studied the flow in axisymmetric abrupt contractions with $\Lambda=2$. In the limit of low Reynolds numbers their results show that $L_{p} / a \sim 0.7$ in accordance with the values in Table 1 for the lowest Reynolds number. This confirms that $L_{p}$ is mainly controlled by the height of the stent strut as already mentioned in Bénard (2005). Even though $L_{p}$ and $L_{d}$ are of the same order of size, the way these recirculation cells affect the wall shear stress is different. The proximal re-circulation cell disturbs the flow less markedly. At a distance equal to $7 a$ to the left of the strut, the wall shear stress recovers the value for a bare artery whereas in the distal region,

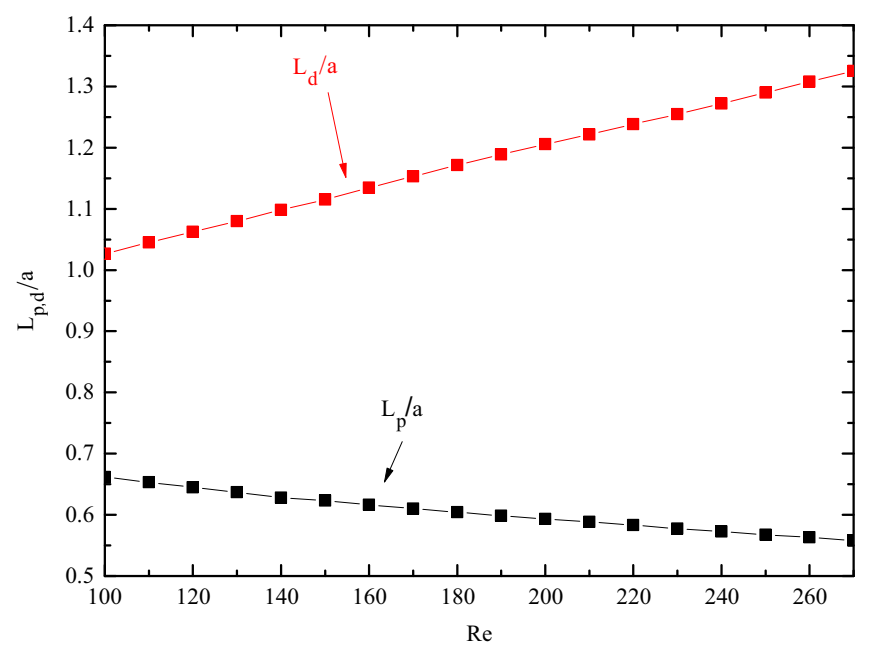

Fig. 6. Distal and proximal re-circulation lengths versus Re (steady case).

Table 1

Comparison of the re-circulation lengths (steady case).

\begin{tabular}{|c|c|c|c|c|c|c|c|c|}
\hline \multirow[t]{2}{*}{$\operatorname{Re}$} & \multicolumn{4}{|l|}{$L_{p} / a$} & \multicolumn{4}{|l|}{$L_{d} / a$} \\
\hline & 106 & 141 & 265 & 282 & 106 & 141 & 265 & 282 \\
\hline Bénard $(\Lambda=17.5)$ & 0.677 & 1 & 0.591 & 1 & 1.118 & 1 & 1.321 & 1 \\
\hline $\begin{array}{l}\text { Kolachalama } \\
\qquad(\Lambda=15)\end{array}$ & 1 & 0.399 & 1 & 0.339 & l & 0.943 & 1 & 1.255 \\
\hline $\begin{array}{l}\text { Present study } \\
\qquad(\Lambda=17.5)\end{array}$ & 0.647 & 0.620 & 0.560 & 0.546 & 1.067 & 1.087 & 1.312 & 1.333 \\
\hline $\begin{array}{l}\text { Relative difference } \\
(\%)\end{array}$ & 4.4 & 55.4 & 5.2 & 61.1 & 4.6 & 15.3 & 0.7 & 6.2 \\
\hline
\end{tabular}


the wall shear stress recovers the undisturbed value at a distance of $44 a$. This result shows the large influence of the distal recirculation on the flow downstream and the lesser effect of the proximal cell on the flow upstream. We now turn our attention to the case of the unsteady flows.

\section{Unsteady flows}

Fig. 7 compares $L_{p}$ and $L_{d}$ given by the PM, SPM and CPM. The main observation is that the difference between the two unsteady models is small (this implies that in the case of drug-eluting stents, the SPM and the CPM would give similar mass fluxes). The maximum relative difference for both lengths $L_{p}$ and $L_{d}$ calculated by the SPM and the CPM is at most 3\% and the time shift observed in Fig. 4 has disappeared. This suggests that $L_{p}$ and $L_{d}$ are no longer sensitive to the details of the velocity profiles at the inlet of the domain and that the boundary layer development around the strut is almost achieved. $L_{p}$ and $L_{d}$ oscillate around the mean values previously obtained in the steady case and deviate at most by $15 \%$ and $17 \%$ respectively. The use of the PM to model the flow in the vicinity of the stent seems to be less questionable as in the case of bare artery. The distal re-circulation length peaks at $L_{d}=1.33 a$ around $t=0.4 \mathrm{~s}$ (maximum of the diastole) and simultaneously the proximal re-circulation length bottoms at $L_{p}=0.53 a$. Fig. 7 clearly shows the strong correlation between the evolution of $L_{p}$

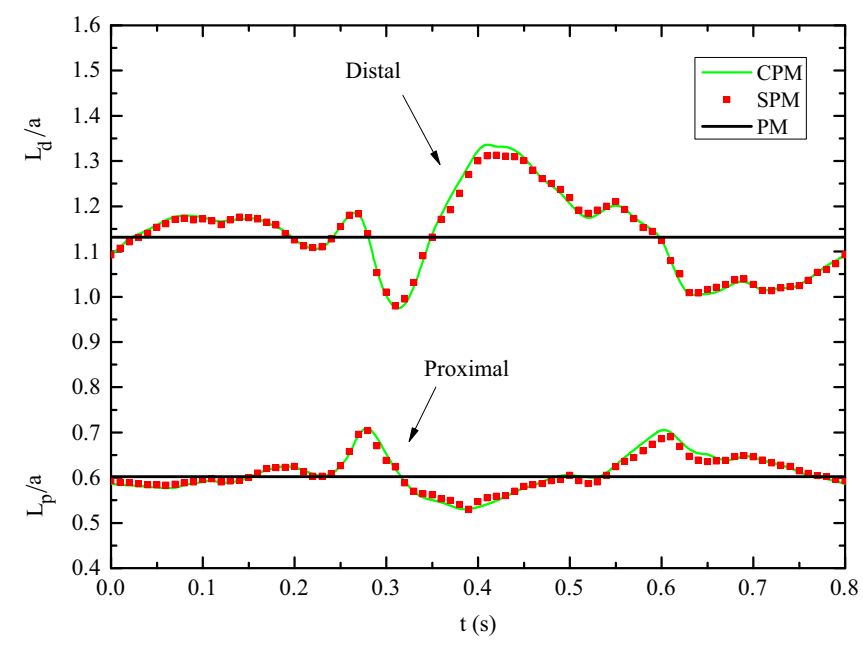

Fig. 7. Re-circulation lengths upstream and downstream of the stent as a function of time

a

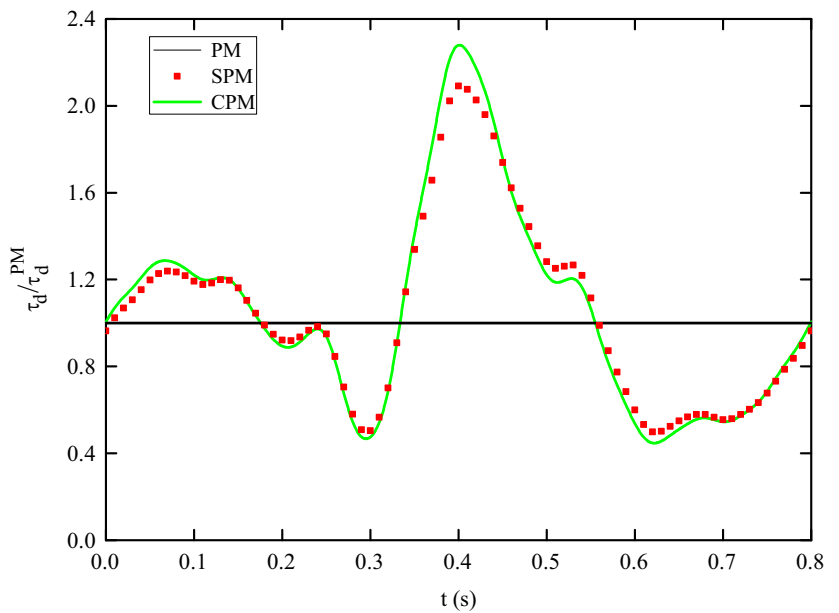

and $L_{d}$ (an increase in $L_{d}$ is related to a decrease in $L_{p}$ and conversely). We also note that the variation in the amplitude of $L_{d}$ is again larger than for $L_{p}$ :

$\frac{L_{d, \text { max }}-L_{d, \text { min }}}{L_{p, \text { max }}-L_{p, \text { min }}} \approx 2$

In the range of Reynolds numbers studied here, $L_{d}$ varies twice as much as $L_{p}$. In other words, the proximal re-circulation cell is less affected by the pulsatility of the flow than the distal one because of the same blockage effect mentioned in the previous steady case.

The size and intensity of these re-circulation cells have also a direct impact on the value of the shear stress (and on the mass flux in the case of drug-eluting stents). Fig. 8 shows the wall shear stress normalized by the steady-state value at a distance $a / 2$ from the strut to the left in the proximal region and to the right in the distal region. Once again, we observe that the SPM and the CPM give similar results. The phase shift present in Fig. 4 has vanished (boundary layer development). The main difference between the SPM and the CPM now resides in the amplitude. The maximum relative difference between the wall shear stress given by the SPM and the CPM in the distal region is at most 13\% and occurs at $t=0.6 \mathrm{~s}$ (minimum of the diastole). In the proximal zone, the maximum relative difference between the two unsteady models is lower (about $8 \%$ ) and still occurs at $t=0.6 \mathrm{~s}$. In a predictable way the PM is also insufficient to assess the wall shear stress in the recirculation cells (for drug-eluting stents the mass flow would also be inaccurate, not including the enhancement of the mass flux by pulsatility). In the distal region, the maximum relative difference between the PM and the SPM is of the order of $120 \%$ while in the proximal region, it does not exceed $30 \%$. As mentioned above, the wall shear stress in the re-circulation cells is drastically smaller than at large distances from the strut. This is an important feature because in the restenosis phenomenon, the response of the arterial wall cells is clearly related to the wall shear stress. The value $\tau_{w}=0.5 \mathrm{~Pa}=5 \mathrm{dyne} / \mathrm{cm}^{2}$ is often cited in the literature (Malek and Izumo, 1996; Malek et al., 1999) to correspond to the threshold under which the smooth muscle cells proliferate. To complete these comparisons, we calculated the ratio between the wall shear stresses in the re-circulation zones (always at distance $a / 2$ from the strut) and those far upstream and downstream of the stent $\tau_{p}(\infty)$ and $\tau_{d}(\infty)$. The wall shear stress is reduced by 6 in the distal re-circulation and by 13.5 in the proximal region for the PM. These values give the order of magnitude of the decreases in the wall shear stress near the stent. For both unsteady models, the

b

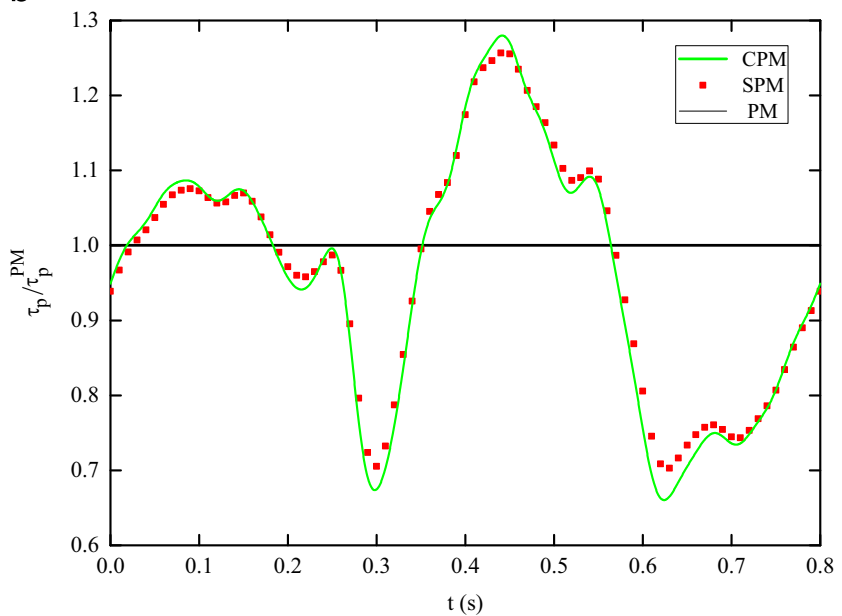

Fig. 8. Wall shear stresses in the vicinity of the strut for the three models 
decrease in the re-circulation cells relative to their values far from the strut fluctuate around the average values obtained previously with $4.5<\tau_{d}(a / 2) / \tau_{d}(\infty)<8$ in the distal region and $8<\tau_{p}(-a / 2) / \tau_{p}(\infty)<22$ in the proximal zone. Compared to the wall shear stress values far from the stent in Fig. 4 , it is obvious that the threshold of $\tau_{w}=0.5 \mathrm{~Pa}$ is reached in the re-circulation cells and particularly in the proximal region (considering only the data given by the PM, the proximal wall shear stress bottoms at $\tau_{p}(-a / 2)=\tau_{p}(\infty) / 13.5 \approx 0.09 \mathrm{~Pa}$ and can even be smaller for both unsteady models).

\section{Computational time}

Another important parameter is the computational time which is often the limiting factor in CFD. In the present study we used the parallel version (4 nodes) of the software and performed the simulations on a quadcore Xeon E5-1607 processor and 8 Gb of DDR RAM. It is irrelevant to compare the computational time for the steady case to its counterparts for the unsteady cases because the former is evidently less demanding (for the sake of information, the computational time of the PM is of the order of $4 \mathrm{~min}$ ). For the SPM and $\mathrm{CPM}$, the computational time was chosen as the time to perform $5 \Delta t=5 \cdot 10^{-3} \mathrm{~s}$. The computational time for the CPM (50 min) is 5 times longer than for the SPM. This five-fold difference is mainly due to the much more complex UDF in the CPM and particularly to the additional efforts to compute the Kelvin and Bessel functions. If we consider only the flow in a bare artery, the additional time in the CPM is not superfluous in view of the results presented in Section 4.1. In the case of the flow past a stent, the situation is less critical and the SPM is an acceptable model if one only focuses on the flow around the stent. In the case of drug eluting stents, the use of the SPM would be even more justified: the characteristic time for the drug diffusion over the distance $a=10^{-4} \mathrm{~m}$ is of the order of $\tau_{m}=a^{2} / D \approx 100 \mathrm{~s}$ (with $D \approx 10^{-10} \mathrm{~m}^{2} / \mathrm{s}$ the mass diffusivity). Comparing this time to the characteristic times of the present flow $\tau=T=0.8 \mathrm{~s}$ and of momentum diffusion $\tau_{v}=a^{2} / \nu \approx 3.10^{-3} \mathrm{~s}$ (with $\nu=\mu / \rho \approx 3.10^{-6} \mathrm{~m}^{2} / \mathrm{s}$ ), we see that the transport phenomena take place on very different time scales. The slowest phenomenon is of controlling the problem, we understand why the PM is often used in the literature.

\section{Conclusion}

In this work we studied the flows given by three hemodynamic models used in the numerical simulation of intra-stent flows. The first model is the Poiseuille profile, widely used in the literature for its simplicity, low computational time and ease to implement. The second model is an unsteady parabolic velocity profile whose mean velocity is taken as the actual average velocity of the real blood flow. Finally the last model is based on the analysis of Womersley. The implementation of this model is not as straightforward as the two others because it requires the computation of complex Bessel and Kelvin functions. From the perspective of its application to the problem of intra-stent flows, the PM is not surprising clearly questionable because the re-circulation lengths and the related values of the wall shear stress are not accurately estimated. The SPM and the CPM give close results, except at times where the peaks of the flow rate occur, but the discrepancies are relatively small (13\% at most). Concerning the computational time, the PM is naturally the least time-consuming. Comparing the SPM and the CPM, we found that the former is at least 5 times more rapid than the latter. Considering the acceptable differences in the results between these unsteady models and the additional computational time of the CPM, we recommend the SPM for future numerical studies of stented arteries.

\section{Conflict of interest statement}

The authors declare that there are no conflicts of interest associated with this research article.

\section{References}

Armaly, B.F., Durst, F., Pereira, J., Schönung, B., 1983. Experimental and theoretical investigation of backward-facing step flow. J. Fluid Mech. 127, 473-496.

Barakat, A.I., Cheng, E.T., 2000. Numerical simulation of fluid mechanical disturbance induced by intravascular stents. In: Proceedings of ICMMB-11: International Conference on Mechanics in Medicine and Biology.

Bénard, N., 2005. Analyse de l'écoulement physiologique dans un stent coronarien : Application à la caractérisation des zones de resténose pariétale (Ph.D. thesis). Université de Poitiers.

Bozsak, F., Chomaz, J.-M., Barakat, A.I., 2014. Modeling the transport of drugs eluted from stents: physical phenomena driving drug distribution in the arterial wall. Biomech. Model. Mechanobiol. 13 (2), 327-347.

Chen, Y., Yan, F., Jiang, W.-T., Wang, Q.-Y., Fan, Y.-B., 2014. Numerical study on effects of drug-coating position of drug-eluting stents on drug concentration. J. Med. Biol. Eng. 34 (5), 487-494.

Coppola, G., Caro, C., 2009. Arterial geometry, flow pattern, wall shear and mass transport: potential physiological significance. J. R. Soc. Interface 6 (35), 519-528.

Grosse, S., Burgmann, S., Schröder, W., 2008. Time-resolved measurements of oscillating flow in a non-collapsing flexible pipe. In: 14th Symposium on Applications of Laser Techniques to Fluid Mechanics, Lisbon, Portugal.

Kastrati, A., Mehilli, J., Pache, J., Kaiser, C., Valgimigli, M., Kelbæk, H., Menichelli, M., Sabaté, M., Suttorp, M.J., Baumgart, D., 2007. Analysis of 14 trials comparing sirolimus-eluting stents with bare-metal stents. New Engl. J. Med. 356 (10), 1030-1039.

Kim, T.D., Seo, T.W., Barakat, A.I., 2004. Numerical simulations of fluid mechanical interactions between two abdominal aortic branches. Korea-Aust. Rheol. J. 16 (2), 75-83.

Kolachalama, V.B., Tzafriri, A.R., Arifin, D.Y., Edelman, E.R., 2009. Luminal flow patterns dictate arterial drug deposition in stent-based delivery. J. Control. Release 133 (1), 24-30.

LaDisa Jr, J.F., Olson, L.E., Molthen, R.C., Hettrick, D.A., Pratt, P.F., Hardel, M.D., Kersten, J.R., Warltier, D.C., Pagel, P.S., 2005. Alterations in wall shear stress predict sites of neointimal hyperplasia after stent implantation in rabbit iliac arteries. Am. J. Physiol.-Heart Circul. Physiol. 288 (5), H2465-H2475.

Malek, A.M., Alper, S.L., Izumo, S., 1999. Hemodynamic shear stress and its role in atherosclerosis. J. Am. Med. Assoc. 282 (21), 2035-2042.

Malek, A.M., Izumo, S., 1996. Mechanism of endothelial cell shape change and cytoskeletal remodeling in response to fluid shear stress. J. Cell Sci. 109 (4), 713-726.

McDonald, D.A., 1955. The relation of pulsatile pressure to flow in arteries. J. Physiol. 127 (3), 533-552.

Mehran, R., Dangas, G., Abizaid, A.S., Mintz, G.S., Lansky, A.J., Satler, L.F., Pichard, A.D., Kent, K.M., Stone, G.W., Leon, M.B., 1999a. Angiographic patterns of instent restenosis classification and implications for long-term outcome. Circulation 100 (18), 1872-1878.

Mehran, R., Dangas, G., Mintz, G.S., Waksman, R., Hong, M.K., Abizaid, A., Abizaid, A.S., Kornowski, R., Lansky, A.J., Laird, J.R., et al., 1999b. In-stent restenosis: the great equalizer: disappointing clinical outcomes with all interventional strategies. J. Am. Coll. Cardiol. 33 (Suppl A), 63A.

Merrill, E.W., 1969. Rheology of blood. Physiol. Rev. 49 (4), 863-888.

Murray, C., Lopez, A., et al., 2002. The world health report 2002: reducing the risks, promoting healthy life. Technical Report, World Health Organization.

O'Brien, C.C., Kolachalama, V.B., Barber, T.J., Simmons, A., Edelman, E.R., 2013. Impact of flow pulsatility on arterial drug distribution in stent-based therapy. J. Control. Release 168 (2), 115-124.

Oliveira, M.S., Oliveira, P.J., Pinho, F.T., Alves, M.A., 2007. Effect of contraction ratio upon viscoelastic flow in contractions: the axisymmetric case. J. Non-Newton. Fluid Mech. 147 (1), 92-108.

Ozolanta, I., Tetere, G., Purinya, B., Kasyanov, V., 1998. Changes in the mechanical properties, biochemical contents and wall structure of the human coronary arteries with age and sex. Med. Eng. Phys. 20, 523-533.

Pielhop, K., Klass, M., Schröder, W., 2015. Experimental analysis of the fluidstructure interaction in finite-length straight vessels. Eur. J. Mech. B/Fluids 50, $71-88$.

Press, W.H., Teukolsky, S.A., Vetterling, W.T., Flannery, B.P., 2007. Numerical Recipes: The Art of Scientific Computing, Third Edition Cambridge University Press, New York.

Seo, T., Schachter, L.G., Barakat, A.I., 2005. Computational study of fluid mechanical disturbance induced by endovascular stents. Ann. Biomed. Eng. 33 (4), 444-456.

Watson, G.N., 1995. A Treatise on the Theory of Bessel functions, Second Edition Cambridge University Press, Cambridge.

Weiler, J.M., Sparrow, E.M., Ramazani, R., 2012. Mass transfer by advection and diffusion from a drug-eluting stent. Int. J. Heat Mass Transf. 55 (1), 1-7. 
Wentzel, J.J., Krams, R., Schuurbiers, J.C., Oomen, J.A., Kloet, J., van der Giessen, W.J., Serruys, P.W., Slager, C.J., 2001. Relationship between neointimal thickness and shear stress after wallstent implantation in human coronary arteries. Circulation 103 (13), 1740-1745.

Williams, D.O., Holubkov, R., Yeh, W., Bourassa, M.G., Al-Bassam, M., Block, P.C., Coady, P., Cohen, H., Cowley, M., Dorros, G., et al., 2000. Percutaneous coronary intervention in the current era compared with 1985-1986 the national heart, lung, and blood institute registries. Circulation 102 (24), 2945-2951.

Womersley, J.R., 1955. Method for the calculation of velocity, rate of flow and viscous drag in arteries when the pressure gradient is known. J. Physiol. 127 (3), 553-563. 